\title{
Cuerpo marginal desde los paratextos literarios de la obra de Con pasión absoluta de Carol Zardetto
}

\author{
Laura Zúñiga-Hernández * \\ https://orcid.org/0000-0003-3099-7369
}

Recibido: 18 de junio de 2020 • Aceptado: 20 de noviembre de 2021

\section{Resumen}

La conformación de un discurso literario va más allá de la narrativa o versificación, en esta se abre una serie de posibilidades a partir de los recursos estilísticos utilizados para enriquecer cada palabra. Así, en las obras, la presencia de paratextos establece una relación entre ellos, su contexto, contenido y encontrar nuevas ideas e interpretaciones.

Por tal motivo, el siguiente análisis aborda los paratextos de la obra Con pasión absoluta $^{1}$ de Carol Zardetto para establecer una conexión metafórica desde los cuerpos marginales como una temática en los textos centroamericanos recientes y que trazan líneas de estudio alternativas a las ya establecidas.

Palabras clave: Violencia, revolución, literatura centroamericana, narrativa.

\footnotetext{
* Maestranda en Literatura Latinoamericana en la Universidad de Costa Rica (UCR), de Costa Rica. Estudios en la Enseñanza de la literatura y el castellano, en la UCR. Diplomado en Educación Primaria, en la Universidad Americana, de Costa Rica. Docente de secundaria y escritora. Correo: langelezluna@gmail.com

${ }^{1}$ Carol Zardetto, Con pasión absoluta (Guatemala: F y G Editores, 2005).
} 


\title{
Marginal body from the literary paratexts of the work, Con pasion absoluta (With Absolute Passion) by Carol Zardetto
}

\begin{abstract}
The composition of a literary discourse goes beyond the narrative or versification. It opens a series of possibilities drawn from stylistic resources used to enrich each word. In that way, in this literary work, the presence of paratexts establishes a relationship among them, their context, content and finding new ideas and interpretations.

For this reason, the following analysis addresses the paratexts of the work, Con pasion absoluta (With Absolute Passion) by Carol Zardetto, in order to establish a metaphoric connection from marginal bodies as a theme in recent Central American texts, and trace alternative lines of study than those which are already established.
\end{abstract}

Key words: Violence, revolution, Central American literature, narrative.

\section{Le marginal dès les paratextes littéraires de l'œuvre Avec passion absolue de Carol Zardetto}

\section{Résumé}

La conformation d'un discours littéraire va au-delà du récit ou la versification; dans cette conformation, une série de possibilités s'ouvre à partir des ressources stylistiques utilisées pour enrichir chaque mot. C'est ainsi que dans les œuvres, la présence des paratextes les met en rapport entre eux, leur contexte et leur contenu en permettant aussi de trouver de nouvelles idées et interprétations.

C'est pourquoi, l'analyse suivante traite les paratextes de l'œuvre Con pasión absoluta (Avec passion absolue) de Carol Zardetto afin d'établir une connexion métaphorique à partir du marginal comme une thématique dans les textes centraméricains récents qui trace aussi des lignes de recherche alternatives à celles qui sont déjà définies.

Mots-clés: Violence, littérature centraméricaine, narrative. 


\section{Introducción}

Los temas en la literatura centroamericana en la década de 1990 y post noventa varían con respecto de las décadas anteriores, uno de los contenidos más recurrentes de análisis es el del cuerpo. Ya lo menciona Le Breton ${ }^{2}$, cuando explica en Antropología del cuerpo y modernidad, cómo el ser humano se simboliza a partir de su corporeidad, pero que en el orden moderno la interpretación varía con respecto a otras épocas. Actualmente se da una ruptura del sujeto, se le relaciona con la búsqueda del uso de las medicinas para controlar las dolencias, se habla de la sensibilidad que lo habita y el signo que lo representa.

Dicha problematización, que se extiende más allá de la definición aportada por el mundo occidental, lleva consigo aspectos que ya Foucault ${ }^{3}$ había tratado anteriormente, el cuerpo es violentado por diversos mecanismos represivos, que le permite al poder el sometimiento de otros. Las estructuras deciden quiénes son marginales, a quiénes se les otorga el carácter de rechazado social: ancianos, enfermos, personas con capacidades limitadas, incluso «lo normal y anormal», están dispuestas por unos pocos y a partir de lo que significa su corporalidad para su entorno social.

En Centroamérica, después del proyecto revolucionario de la década de 1980, ya en decadencia y desencanto, se consolida lentamente el eje de lo íntimo desde el cuerpo; por lo cual, al entrar en décadas recientes, el escritor vuelve su mirada hacia lo personal, intimista, apartando las aspiraciones derrocadas de los antecesores. Se vuelve un proceso de consolidación de nuevos paradigmas, asumidos desde las individualidades marginales, sumisas y violentadas tras la utopía revolucionista.

Dentro de la contemporaneidad explicada por $Z_{a v a l a}^{4}$, se revela la búsqueda constante de técnicas que innoven el texto literario; parte de estos abordajes no solo se dan en la narrativa, sino en poemarios; de este modo, la implementación de la metáfora se establece en su relación con el significado de la nación y en cómo se reconstruye después de que cayera la revolución centroamericana de la década de 1980. Así, los sentidos metafóricos demuestran que los cuerpos individuales personifican la violencia, mutilación y el desencanto del pasado en las sociedades sufrientes de la década de 1980. Por lo tanto, el siguiente análisis pretende explicar, a partir de los paratextos de la obra Con pasión absoluta de Zardetto ${ }^{5}$, la representación metafórica de la corporeidad marginal presente en el texto. Sin duda, la escogencia de esta

\footnotetext{
${ }^{2}$ David Le Breton, Antropología del cuerpo y modernidad (Buenos Aires: Ediciones Nueva Visión, 2002).

${ }^{3}$ Michelle Foucault, Vigilar y castigar. Nacimiento de la prisión (México: Siglo XXI editores, 1976).

${ }^{4}$ Magda Zavala, Globalización y literatura en América Central: escritores y editoriales. Hacia una historia de las literaturas centroamericanas-Intersecciones y trasgresiones: Propuesta para una historiografia literaria en Centroamérica (Guatemala: F y G Editores, 2008).

${ }^{5}$ Zardetto, Con pasión absoluta...
} 
obra se basa en la influencia que dicha novela tiene sobre la literatura actual centroamericana, ganadora de un premio en el 2004; aborda temas históricos, de la memoria y la violencia, encarnados desde la mirada femenina que, mediante diversos recursos, lingüísticos y paratextuales, demuestra la hibridez de las nuevas obras de la región, así como la reescritura de la historia. La novela de Zardetto, publicada en 2005, trata de Irene, quien decide regresar a su país natal porque su abuela está enferma. Al volver, se encuentra con una Guatemala diferente y con una memoria colectiva que llena todos los espacios tras varias décadas de diversos conflictos y cambios. Este reencuentro con su pasado y lugar de nacimiento, le permite reconstruir su propia historia, narrada desde la mirada femenina que atraviesa tres generaciones: la de Irene (la protagonista), Toya y Nena, la abuela y la madre de la protagonista, respectivamente.

Se entrecruzan aquí los frágiles límites del recuerdo y los sucesos históricos con la ficción, hecho muy característico de la literatura centroamericana contemporánea, como una forma de cicatrizar el dolor y los traumas, un método para saldar la deuda ideológica y reconstruir la historia de un país fragmentado por las consecuencias catastróficas -a nivel social, político, económico y psicológico- de la guerra.

\section{Contexto histórico}

La obra de Zardetto se desarrolla desde un contexto de violencia, la cual es acarreada a lo largo de décadas en Guatemala y en toda Centroamérica. Hacia finales de la década de 1970, la región centroamericana había estado en situaciones convulsas, tales como las crisis económicas que alcanzan déficits muy altos en la región; a su vez, se forman movimientos populares como expresión de la lucha de clases. Maldonado ${ }^{6}$ explica con detalle la situación particular del país, con un desarrollo desigual, marcado por hechos políticos, sociales y culturales; influencia de etnias diferentes, variadas lenguas que van desde el español hasta el mayense.

Desde el periodo de la independencia se daban estas desigualdades, provenientes de las clases sociales, lo que provocó, por mucho tiempo, el aislamiento de la población indígena. Con la independencia, se trató de eliminar esta situación; sin embargo, no se obtuvo el objetivo, pues los grupos de poder ejercieron disputas para conservar los antiguos hábitos.

Más adelante, en la primera mitad del siglo XIX, se inician periodos dictatoriales y regímenes autoritarios en los cuales prevalece la persecución a la Iglesia católica, principalmente porque estaba enlazada con grupos

\footnotetext{
${ }^{6}$ María del Pilar Maldonado, «Sobre la memoria cultural e histórica en Guatemala. La obra de Carol Zardetto: Con pasión absoluta» (tesis doctoral, Universität Wien, 2010), acceso: 27 de mayo de 2021, https://docplayer.es/14309755-Diplomarbeit-titel-der-diplomarbeit-sobre-la-memoria-cultural-ehistorica-en-guatemala-la-obra-de-carol-zardetto-con-pasion-absoluta.html
} 
indígenas que lucharon durante varios años para reivindicar sus derechos, lo que un tiempo después provoca su incorporación a la lucha armada; además, rige el menosprecio por la clase campesina, ya que ante todo, los gobernantes querían la modernización del país.

Hay otros hechos que van esculpiendo la identidad guatemalteca, entre ellos se resalta la explotación del cultivo y comercio del café, con esta se evidenció, aún más, el beneficio obtenido por las clases altas; también, se dio un incremento significativo de la introducción del cultivo del banano y de empresas estadounidenses.

Entre las décadas de 1960 y 1980, inicia un espacio violento en la región centroamericana; en el caso de Guatemala, se dan enfrentamientos por cuestiones políticas, económicas, sociales y de etnias; de este modo, el gobierno usa el poder mediante la militarización de los conflictos para dar resolución a las múltiples manifestaciones y en consecuencia se da la lucha contra los sectores insurgentes.

Fraudes electorales, secuestros, terrorismo, discriminación, pobreza y muertes debido a las luchas sociales por defender tierras, identidad y justicia social a nivel económico, fueron detonantes para protestas, además se dio una falta de apoyo internacional y la conformación de grupos distintos en disputa del poder.

Para los 2000, el panorama guatemalteco no varía y se sigue dando la corrupción, el narcotráfico y la intrusión de grupos informales de poder, tales como las maras; estas últimas empobrecen y violentan más el territorio y, sobre todo, aumenta la inseguridad ciudadana, situaciones que llegan hasta el presente.

\section{Estado de la cuestión}

Hay investigaciones que tratan sobre las temáticas de este artículo, tales como el cuerpo, la metáfora, los paratextos, las cuales serán expuestas brevemente a continuación.

En primer lugar, la imagen del cuerpo como Centroamérica es, en sí, una metáfora, una representación implícita en muchos textos. Su perspectiva sobrepasa lo colectivo y se enfoca en una individualidad que expresa un espacio macro y micro, pero intimista. Le Breton ${ }^{7}$ ya lo decía: «[...] El cuerpo funciona como un límite fronterizo que delimita ante los otros, la presencia del sujeto. Es factor de individuación». La distinción que se hace con los otros a partir del cuerpo implica ser parte de un todo, un grupo en el que se diluye el individuo en medio del cosmos. En la modernidad, este representa una

${ }^{7}$ Ibíd., 22. 
posesión en el que se puede hacer, se construye, se deforma, se cura, enferma o se violenta.

En obras recientes, se figura el cuerpo como en la novela $A-B$ sudario $^{8}$ de Jacinta Escudos, quien con tono autobiográfico habla de la exploración del sujeto y su represión desde la individualidad; asimismo, en la obra se frecuentan espacios para el cuerpo, puesto que al final este se convierte en un espacio delimitado por sí mismo.

Sublimes y perversos de Lety Elvir ${ }^{9}$, por su parte, explica dos estéticas distintas de la corporalidad, es decir, la de lo sublime y lo perverso. La obra problematiza las relaciones entre mujer, sexualidad y escritura, los cuerpos pasan de ser objetos a sujetos. Las representaciones de lo masculino y femenino cambian, se da una evidente liberación de los parámetros sociales, las reglas se rompen, hay un espacio para la experimentación y la experiencia. Otro ejemplo es De fronteras $^{10}$, de Claudia Hernández, quien con una serie de relatos de corte realista y fantástico, interpreta la visión de la región centroamericana enmarcada, principalmente, desde la voz de la mujer migrante y la denigración de su cuerpo por parte de la hegemonía circundante. En segundo lugar, sobre los paratextos existen varias investigaciones que los explican desde su definición y funcionamiento. Álamo ${ }^{11}$ los define desde los principios de Genette y clasifica los diferentes elementos que componen un texto, o como él lo denomina, «transtextuales», divididos en cinco: intertextualidad, paratextualidad, metatextualidad, hipertextualidad y architextualidad. Además de las definiciones desarrolladas, se establece la relación con respecto al ámbito editorial y los elementos constituyentes del diseño, es decir, «[...] capítulo (epígrafe, parágrafo o párrafo); dedicatoria (dedicatoria intradiegética); explicit/incipit; prólogo (exordio) o proemio/ epílogo; título (epónimo, subtítulo)» ${ }^{12}$.

Castro $^{13}$ también relaciona el término de lo paratextual en un estudio historiográfico de dos textos que tratan sobre lo indígena, uno costarricense y otro guatemalteco. La autora se vale de estos para explicar la hibridez presente, los datos brindan validez a lo tratado en las narraciones, ambas provienen de un yo que cuenta desde el no sujeto indígena.

\footnotetext{
${ }^{8}$ Jacinta Escudos, AB sudario (San Salvador: Alfaguara, 2003).

${ }^{9}$ Lety Elvir, Sublimes y perversos (Honduras: Litografía López, 2005).

${ }^{10}$ Claudia Hernández, De fronteras (Guatemala: Editorial Piedra Santa, 2007).

${ }^{11}$ Francisco Álamo, «Paratextualidad y novela: Las partes del texto o el diseño editorial», Anuario de estudios filológicos 32, (2009): 5-21.

12 Ibíd., 11.

${ }^{13}$ María de los Ángeles Castro, «La construcción de lo indígena en textos etnoficcionales en Centroamérica», Ístmica, n. ${ }^{\circ} 11$ (2007): 131-154.
} 
Con respecto de la metáfora, Fajardo ${ }^{14}$ explica que se refiere al uso del lenguaje figurado que debe ser interpretado por el lector; es decir: «[...] son todas aquellas expresiones en las que tenemos que ubicar dentro de una gama de posibles sugerencias y que se acomodan más al contexto; el lenguaje metafórico, se dice, tiene un efecto contaminante que impide el razonamiento científico y objetivo.» ${ }^{15}$

\section{Conceptualización}

\section{La marginalidad}

Seguidamente se detallará, con brevedad, el concepto de la marginalidad. Gama $^{16}$ explica que la existencia de lo marginal se da, primero, al haber algo catalogado como «normal», es decir, la presencia del otro, ese que no es como el sujeto que habla. El patrón de normal y anormal expresa, en consecuencia, un asunto de poder, una posición con respecto al subordinado.

El sujeto marginal, en la literatura centroamericana, se concibe desde el panorama que queda después de la guerra y los acuerdos de paz. Arias ${ }^{17}$ expone que el sujeto centroamericano sobrevivió, pero no fue salvado ni se redimió con las batallas entabladas, continuó sumido en la pena, la desesperación y del todo no convencido de que sus naciones efectivamente lo habían hecho.

Lo anterior, en consecuencia, expone la búsqueda de una identidad, una posición social, un pertenecer más allá de los límites; busca permanecer, a pesar de la «pena y la desesperación». Igualmente, se manifiesta a través de personajes que hasta el momento eran invisibilizados, como ocurre con las mujeres, indígenas o niños.

En resumen, la necesidad de discutir sobre la marginalidad presente en el discurso del istmo es una manera de mostrar la cultura marginada; porque, «desde allá intentan moldear un nuevo ser sobre las ruinas y fragmentos de otros tiempos y otros espacios pues ambos (la novela y el testimonio) son diferentes estrategias de resistencia» ${ }^{18}$. Dicha resistencia es la tónica que cede paso al área de la subjetividad que reestructura a la población y a los nuevos hablantes.

\footnotetext{
${ }^{14}$ Luz Amparo Fajardo, «La metáfora, un recurso en la formación de pensamiento», Instituto de Ciencias de la Educación para la Investigación Interdisciplinaria de la Facultad de Ciencias Humanas, n. ${ }^{\circ} 11$ (2007): 103-111, acceso: 27 de mayo de 2021, https://cerac.unlpam.edu.ar/index.php/praxis/article/view/471

${ }^{15}$ Ibíd., 105.

${ }^{16}$ Michelle Gama, «El cuerpo marginal: los símbolos del cuerpo en el cuento "El cobrador” de Rubem Fonseca», Mitologías hoy, n. ${ }^{\circ} 6$ (2014): 48-55. acceso: 27 de mayo de 2021, https://revistes.uab.cat/mitologias/article/view/v6-gama

${ }_{17}$ Arturo Arias, Gestos ceremoniales. Narrativa centroamericana 1960-1990 (Guatemala: Artemis y Edinter, 1998), 273.

${ }^{18}$ Ibíd., 317.
} 


\section{El cuerpo}

Quizá este sea uno de los elementos más relevantes de entender en la literatura centroamericana, pues es un espacio social e individual. $\mathrm{Cortez}^{19}$ ya lo había explicado acerca de los cuentos de Claudia Hernández: ¿Dónde se puede llevar esta culpa sino en el cuerpo mismo? La marca en la corporeidad se da en los violentados, mutilados o sometidos a un poder, aspectos propios de la realidad de Centroamérica durante décadas, donde Guatemala no es la excepción.

Sesarego ${ }^{20}$ expone que la presencia y la ausencia del cuerpo se distingue por su nivel sensitivo y fenomenológico. En el primero, se desata toda la información recibida mediante la percepción, tanto de manera interna como externa: temperatura, dolor, postura, entre otros. El segundo se percibe solo en ciertos momentos, como en la enfermedad o la dolencia. Asimismo, el autor lo explica desde tres dominios: el flujo de conciencia en sí mismo, el ambiente y la presencia corporal; donde, según él, este último es el más relevante, ya que en la mayor parte del cuerpo su presencia es invisible, incluso la llama «marginal», y en otros momentos se es consciente de ella, porque se necesita que así lo sea, como cuando se quiere aprender alguna labor que lo involucre directamente o en el caso de las enfermedades.

Le Breton ${ }^{21}$ abre otra serie de conceptualizaciones e indica cómo se ha entendido a lo largo del tiempo; hace hincapié en el simbolismo que posee dentro de cada sociedad específica, en la que muchas veces se le define desde un producto cultural nombrado hasta sus aspectos biológicos.

Las simbolizaciones hechas por el autor también giran en torno de la corporeidad como un espacio individual separado de los otros: «La noción moderna de cuerpo es un efecto de la estructura individualista del campo social, una consecuencia de la ruptura de la solidaridad que mezcla la persona con la colectividad y con el cosmos a través de un tejido de correspondencias en el que todo se sostiene» ${ }^{22}$.

En conclusión, el cuerpo se define por los individuos a partir de las relaciones con los otros; sin embargo, son individuales, aunque su entorno sea de una colectividad en la que se desarrolla. Por lo tanto, funciona como un límite fronterizo entre los demás y radica la presencia del sujeto.

\footnotetext{
${ }^{19}$ Beatriz Cortez, Estética del cinismo. Pasión y desencanto en la literatura centroamericana de posguerra (Guatemala: F y G Editores, 2010).

${ }^{20}$ Sesarego, Roberto, «El cuerpo marginal: aspectos de la fenomenología del cuerpo explorada desde Gurwitsch», conferencia pronunciada en la I Jornadas de Estudiantes Departamento de Filosofía en la Universidad de Buenos Aires, 25 al 28 de noviembre de 2014.

${ }^{21}$ Ibíd.

${ }^{22}$ Le Breton, Antropología del..., 16. 


\section{Paratextos}

En sí, estos se pueden definir desde su función, como guía para simbolizar el texto, determinan la lectura y la comprensión. Se distinguen dos posiciones: el bloque del autor y el del editor. Según lo afirma Sabia ${ }^{23}$, el primero se relaciona con lo que el autor propiamente utiliza para estructurar su obra, mientras que el segundo responde a las necesidades particulares de edición: lo comercial, la difusión y la distribución.

Genette, citado por $\mathrm{Paz}^{24}$, estudioso de los paratextos, los valora de un modo intermedio entre lo dicho y lo exterior al texto, lo cual concierne al autor y a la editorial. Entre estos se menciona: títulos, subtítulos, prefacios, índices y dedicatorias.

Ahora bien, de los diversos paratextos existentes, los elegidos para este artículo, son los títulos, como enunciados que orientan, anticipan, especifican y designan el contenido del libro o el texto. Se consideran también como una «instancia» comunicativa compuesta de diversos elementos: un mensaje, un destinador (por lo general es el autor) y un destinatario (público, que más bien son los lectores, quienes leen o no por completo el libro, sin embargo, son los receptores).

Equivalentemente, otro elemento son las dedicatorias, las cuales consisten en una práctica en la cual la obra se dedica a una persona, benefactor o institución con el fin de homenajearlo de manera real o ideal. Esta práctica se remonta a la Roma antigua, pasa por la Edad Clásica y llega hasta estos días. Por lo general se ubican al principio del libro, sin embargo, también al final, o bien, en partes del texto ya desarrollado.

Cuenta con elementos tales como el dedicador, que será el autor, aunque también puede ser un traductor. En el caso de los dedicatarios, se clasifican en dos: públicos (persona más reconocida con una relación intelectual o académica con el escritor) y privados (persona con una relación personal con el autor).

En síntesis, de todos los aspectos desarrollados con anterioridad, se destaca el hecho de que los paratextos tienen una función específica y son objetos interpretables en un análisis, pues son una guía para llegar a una explicación de los textos y para la comprensión lectora.

\footnotetext{
${ }^{23}$ Säid Sabia, «Paratexto. Títulos, dedicatorias y epígrafes en algunas novelas mexicanas», Espéculo: Revista de estudios literarios, n. $^{\circ} 31$ (2005), acceso: 27 de mayo de 2021, https://webs.ucm.es/info/especulo/numero31/paratext.html

${ }^{24}$ José María Paz, «Texto y paratexto en el Quijote», conferencia pronunciada en Actas II. II Congreso de la Asociación Internacional Siglo de Oro. Centro Cervantes Virtual, Universidad de Salamanca, 1990, acceso: 3 de junio del 2021, https://cvc.cervantes.es/literatura/aiso/pdf/02/aiso 22 030.pdf
} 


\section{Metáfora}

Este término posee muchas definiciones, de las cuales se mencionarán brevemente algunas. Lakoff y Jonhson ${ }^{25}$ explican que, aunque hay personas que pretendan vivir sin metáforas, estas son parte de la vida, pues se impregnan a nivel del lenguaje, del pensamiento y la acción desde temprana edad. En primera instancia explican que: «La metáfora es un mecanismo de analogía en el que se concibe un concepto que pertenece a un dominio conceptual determinado en función de otro dominio conceptual, y en el que se establecen correspondencias y proyecciones entre los atributos de ambos dominios $»^{26}$.

Es decir, no se puede observar sin ser referida a otros significantes, es la relación entre un término y otro, perteneciente a un dominio conceptual en el que se realizan correspondencias.

Otro autor que trata el tema es Borges; Laín ${ }^{27}$ explica que esta es una cuestión de nombrar «un lugar de» como un sustantivo; se le equipara con un símil o sinécdoque, es decir, un proceso de comparación. Al mismo tiempo, se conceptualiza a partir de la poesía, como la expresión que representa su estado más esencial, utiliza la figura para explotarse a sí misma, lo que le da la cualidad de emplearse cientos de veces más en la voz poética.

Por último, otra concepción la brinda Fajardo ${ }^{28}$, quien explica que esta se refiere al uso del lenguaje figurado, objeto de interpretación por el lector, dado a través de un proceso de posibles connotaciones.

En resumen, cada una de las definiciones de la figura literaria se remiten a procesos de simbolización, de lectura y relaciones que realiza el lector sobre determinados conceptos y que pueden generar nuevos significados.

\section{Análisis de Con pasión absoluta ${ }^{29}$}

Seguidamente se detallará, con brevedad, el concepto de la marginalidad. $\mathrm{Gama}^{30}$ explica que la existencia de lo marginal se da, primero, al haber algo catalogado como «normal», es decir, la presencia del otro, ese que no es como

\footnotetext{
${ }^{25}$ George Lakoff y Mark Jonhson, Metáforas de la vida cotidiana (Madrid: Cátedra Teorema, 1995).

${ }^{26}$ Carolina Boubée, Olga Graciela Delorenzi y Patricia Sastre Vázquez, «La comprensión: proceso lingüístico y matemático», Revista Iberoamericana de Educación 46, n. ${ }^{\circ} 8$ (2008): 1-9.

${ }^{27}$ Guillermo Laín, «Teoría y práctica de la metáfora en torno a Fervor de Buenos Aires, de Borges», Cuadernos de ALEPH, . $^{\circ} 2$ (2007): 79-93.

${ }^{28}$ Fajardo, «La metáfora...

${ }^{29}$ Ibíd.

${ }^{30}$ Michelle Gama, «El cuerpo marginal: los símbolos del cuerpo en el cuento "El cobrador” de Rubem Fonseca», Mitologías hoy, n. ${ }^{\circ}$ (2014): 48-55. acceso: 27 de mayo de 2021, https://revistes.uab.cat/mitologias/article/view/v6-gama
} 
el sujeto que habla. El patrón de normal y anormal expresa, en consecuencia, un asunto de poder, una posición con respecto al subordinado.

Título

Desde el título, Con pasión absoluta, la autora presenta un juego de palabras que, mediante su fonética, permite la interpretación de diversos modos. En primer lugar, se compone en su morfología y función sintáctica, como una preposición, un sustantivo y un adjetivo femenino, es decir, un grupo nominal en el cual es importante el nombre y no tanto las acciones; de acuerdo con Genette $^{31}$, este se puede clasificar como un título temático, pues expresa a qué se va a referir el contenido de la obra y no a un personaje o situación en particular.

En segundo lugar, si se analiza desde la fonética, es una tautología, interpretada como «Con-pasión-absoluta», o bien, entender «compasión absoluta», ambos enunciados dependen de su fonética, realizan un juego que afecta directamente el sentido del texto y su contenido. En el primero, se da la predominancia de las emociones, el sentido de la preferencia por alguien o bien la afición a otra persona; el hecho de que sea absoluta le da un valor maximizado y exclusivo. En cuanto a la acepción de una pasión de ánimo, refleja un efecto desalentador y triste, cercana al contenido del texto de Zardetto.

En el segundo enunciado «compasión absoluta» se comprende como una persona con suficiente amor al prójimo, sumamente desprendida, o bien, siguiendo la acepción de la RAE, «Sentimiento de pena, de ternura y de identificación ante los males de alguien» ${ }^{32}$. Nuevamente, a partir de esta interpretación, el contenido de la obra se refleja de una u otra manera, porque metaforiza en sí la historia guatemalteca.

Recapitulando, tal como se expone, cualquiera de las dos interpretaciones se relaciona con el hecho de entender la novela desde la marginalidad de los personajes, los cuales recuerdan su pasado y presente llenos de nostalgia, tristeza o incluso entusiasmo por los acontecimientos vividos, marcados en sus corporeidades.

\section{Dedicatoria}

La dedicatoria expresa: «A las mujeres que tejieron mi infancia» ${ }^{33}$, como se observa, se entabla un diálogo con un grupo marginado: las mujeres, quienes contarán la historia llena de dolor y cargas ideológicas fuertes; marcadas por el patriarcado.

\footnotetext{
31 Ibíd.

${ }^{32}$ Diccionario de la Real Academia Española, «compasión», última modificación 2014, https://dle.rae.es/pasión?m=form

${ }^{33}$ Zardeto, Con pasión..., 7.
} 
Este paratexto, en primer lugar, se puede definir desde el psicoanálisis, se afirma lo que no es mujer es hombre y viceversa, esta cuestión remite a la posición cultural y social marginada de la mujer, incluso en este siglo, tal como lo explica Santos ${ }^{34}$ :

[...] Si esto es cierto, tenemos que plantear entonces la necesidad de que el psicoanálisis relativice el falocentrismo tanto del inconsciente como de sus teorías y pensar que si en la clínica encontramos que los inconscientes de hombres y mujeres son falocéntricos no es porque la biología así lo determina, como lo pensó Freud, ni es efecto de un orden simbólico ahistórico como lo pretende la teoría lacaniana, sino que es el resultado de procesos culturales cuyas condiciones de producción y reproducción nos corresponde investigar.

Por lo cual, se considerará que la facultad para determinar uno y otro sexo es su físico y mentalidad; pero, sobre todo, el carácter social y cultural existente. Asimismo, como un asunto biológico, la dedicatoria enuncia que no está dedicado a hombres o, por lo menos, esa no es la primera intención de la autora. Lo segundo es que el hecho de ser dedicado a «las mujeres», refuerza la marginalidad, un estatus, donde el género femenino se limita a un nivel inferior social, político, laboral o educativo, dicha situación no es ajena al entorno guatemalteco.

Las mujeres a quienes se dedica el libro, además, no son cualquier tipo de mujer, son «a las que tejieron mi infancia», esta oración subordinada y metafórica, sin duda, enfatiza el sentir de una figura marginal, pues tanto las palabras involucradas, «mujer»y «que tejieron mi infancia», remiten a aspectos meramente condicionados al género, a un carácter corporal y de rol como «tejer», tarea que está implícitamente destinada a las mujeres.

Finalmente, la mención de la infancia muestra un carácter corporal, un paso, un cambio de la niñez a la adolescencia y, esta, a la adultez o, por qué no, a la vejez. Quiere decir que la dedicatoria abre un espacio de encuentro hacia el sentir femenino, su carácter corporal, limitado a los acontecimientos de mujeres que implica un cuerpo traspasado por el espacio social que lo determina, modifica, construye y establece como tal.

\section{Capítulo V}

Este apartado se inicia con los acontecimientos ocurridos en una cárcel, los múltiples asesinatos y la crueldad desatada, contextualiza los hechos en un espacio y periodo en sí. La cárcel es un espacio marginal, destinado a aquellos que son lanzados y a quienes se les condiciona su libertad.

\footnotetext{
${ }^{34}$ Luis Santos, «¿Qué es ser hombre? Reflexiones sobre la masculinidad», en Relación de saberes (Bogotá: Universidad Nacional de Colombia, 1998).
} 
Más adelante se trae a colación el sobrenombre que utilizaron para el país guatemalteco durante la época de las bananeras, es decir, hacia principios del siglo XX, cuando el auge de la siembra y exportación del banano hizo que Centroamérica apareciera en el mundo, incluso en la literatura, gracias al cultivo del banano; no obstante, esta frase sin duda se acuñó de forma despectiva, de lo cual no queda exenta Guatemala, llamada «Banana Republic». La designación da pie para entender lo desarrollado más adelante en el paratexto, donde se evoca la memoria del territorio con párrafos expositivos e históricos concernientes al café y su cultivo.

Otro paratexto consiste en un cuadro de texto separado de la narración, en el cual se menciona: «Barberena, un diminuto pueblo, a medias de un camino polvorienta [...] Punto de referencia de una geografía de muchas maneras. Accidentada- Volcánica sísmica, quizá incluso trágica. Marcada por la huella de Guatemala porque era carne de su carne» ${ }^{35}$. Este episodio contiene varias palabras clave que refuerzan la marginalidad en el texto. Una de ellas es pensar en una frontera, la cual limita a una región determinada. Se da un hecho histórico como el terremoto de 1913, la autora caracteriza a la zona como: volcánica, sísmica y trágica; dicho espacio sufriente se compara con un cuerpo geográfico y social violentado por fenómenos naturales que lo marginan, además de que condicionan a la ciudad a una problemática nacional y económica desventajosa.

El paratexto cierra con una referencia a la corporeidad: «Marcada por la huella de Guatemala porque era carne de su carne» ${ }^{36}$. El participio en función adjetiva de «marcada» evoca a una cicatriz, una marca que dejó el pasado, al mismo tiempo se presenta una huella, no cualquiera, es en «la carne de su carne», con lo cual se remite una vez más al cuerpo, a un conjunto que se representa con una metáfora: la violencia, el sufrimiento del país y sus habitantes.

La tragedia, las marcas y el sufrimiento remiten al cuerpo sufrido, violentado, por tanto marginal, de un pueblo, mientras que la geografía limita su relación con otros mediante una frontera, es decir, de manera corporal.

\section{Segunda parte}

En otro apartado, se desarrollan, en forma de columnas dentro de cuadros, los aportes históricos del café y su cultivo. Este paratexto se establece en cuatro «movimientos», se escribe en un gran paréntesis. Así se conforma una especie de código de validez de la ficción a partir de la memoria histórica y cultural de Guatemala.

\footnotetext{
${ }^{35}$ Zardeto, Con pasión absoluta..., 116.

${ }^{36}$ Ibíd. 
La primera parte está dividida en dos, paralelas una de la otra, con un formato de letra diferente, una en itálica y la otra no. Una columna expone la historia del café y la otra sobre el gobierno dictatorial y déspota, explican sobre el poder, así se puede confirmar quiénes son subordinados y quiénes subordinan; la situación se respalda por aspectos corporales: asesinatos y desaparecidos. Por ejemplo: «[...] quedaban tirados en los caminos por esparcimiento, los cuerpos de pequeños rateros y contrabandistas, arrinconados por el hambre» ${ }^{37}$.

El hecho de contar en paralelo la historia del café, su cultivo, cómo se cosecha y exporta, evidencia lo que debe contarse: la fachada del poder económico, la corrupción y marginación, los cuerpos violentados, usurpados y muertos terminan de exhibir la otra cara de la moneda, la violencia ejercida por los grupos más poderosos de la Guatemala del pasado.

Finalmente, la corporeidad marginal se refuerza con las últimas líneas: «Mi madre, uno de esos seres sin nombre que la historia iba, va dejando al margen. $\rangle^{38} \mathrm{El}$ final es evidente, el cuerpo de la madre engendra y carga en su vientre un ser, representa el cuerpo que cambia y se transforma para dar vida; sin embargo, Zardetto presenta una mujer, «mi madre», sin nombre, es decir, sin una identidad, una persona que no pertenece a un grupo, una historia que termina al margen, fuera de lo trascendente.

\section{Tercera parte}

Por último, los movimientos del uno al cuatro se caracterizan por la apertura y cierre del paréntesis. En el Diccionario de la Real Academia Española, algunas de las trece acepciones que aparecen de la palabra «movimiento» son: «acción y efecto de mover y conjunto de alteraciones o novedades ocurridas durante un periodo de tiempo, en algunos campos de la actividad humana ${ }^{39}$, lo cual se expresa, a lo largo de estos párrafos, con el ir y venir de los personajes.

El primer movimiento narra sobre la protagonista, una niña quien sufre debido a la ausencia-presencia de su padre, el alcalde del pueblo que lleva una vida de libertinaje y sin responsabilidades. En el apartado, se expone el ir y venir de la protagonista en búsqueda del dinero que le entrega su papá para solventar los gastos. En el fragmento hay referencias constantes a la corporeidad, al inicio se mencionan, por ejemplo: «[...] las mujeres gordas y pintarrejeadas $[\ldots]\rangle^{40}$, quienes le hacían gestos de desprecio a la niña, se les caracteriza como «sucias» para referirse a ellas en cuanto a la prostitución.

\footnotetext{
${ }^{37}$ Ibíd., 118-119.

${ }^{38}$ Ibíd., 121.

${ }^{39}$ Diccionario de la Real Academia Española, «movimiento», última modificación 2014, https://dle.rae.es/movimiento

${ }^{40}$ Zardeto, Con pasión absoluta..., 121. 
A su vez, se instauran vínculos corporales mediante la violencia, la madre sufría constantes abusos por parte del alcalde y la niña era rechazada por los empleados de la alcaldía. El frecuente rechazo y abuso se mitiga debido a la condición física del padre, de quien se menciona: "Yo odiaba a mi papá, tal vez porque era tan guapo ${ }^{41}$, como se destaca en la cita, el personaje se basa en el físico para expresar un sentimiento: el de odio hacia el padre.

La corporeidad respalda la figura del poder del padre en su trabajo, capaz de llevar la transformación corporal de la niña que narra cuando la mandaba a buscar y se sentía como un conejo; lo cual refleja la marginalidad de la pequeña, en contraste con el poderío de su papá, la convencen, a tal punto, de presentar el sentimiento de amor-odio hacia él, confirmando, en el paratexto, las diversas corporeidades a nivel emocional y físico.

En el segundo movimiento se narra más la relación de la niña con sus padres y con demás familiares. Hay mención a tradiciones guatemaltecas, brujerías, bodas y sobre el amorío de la madre de la protagonista.

El desencanto amoroso se plasma en todo el apartado, hay constantes reseñas del cuerpo y cómo es descrito, usado y purificado. En primer lugar, se describe algunos personajes masculinos descritos por su físico, por lo general, peyorativas y animalizadas: «[...] parecía un chucho [...]», «Era canillón, esqueletudo, con la cara verde, toda chupada. Feo como él solo ${ }^{42}$, se añade de los pasantes del juzgado un rasgo animal, «como ratas», o bien del padre se dice que es «una bestia».

La madre, al descubrir que su amante se casa con otra, la busca y ambas sufren maltrato físico. De dicha relación frustrada, la madre queda embarazada, lo que la condena al desprecio por parte del esposo. Su condición depresiva se torna a tal punto que empieza con dolencias en el corazón y «[...] sentía que se moría» ${ }^{43}$, la conduce a buscar ayuda con un brujo, quien recurre a remedios corporales para descubrir que tiene sobre sí un hechizo, lanzado por la novia.

Hay muchas referencias sobre lo anterior, por ejemplo: «Le pasó un huevo por el cuerpo» o «Allí bajo la luna se pasaba los manojos de candelas de sebo de siete colores, repitiendo una letanía y después las quemaba en una palangana, donde ardían haciendo una humazón. Agarraba unas ramas de chilca y se somataba con ellas por todos lados $[\ldots] \gg{ }^{44}$.

Una vez más el cuerpo sufre, se purifica, es sacrificado para encontrar la paz y la aceptación, principalmente de la mujer, quien por su error paga las

\footnotetext{
${ }^{41}$ Ibíd., 123.

42 Ibíd., 124.

${ }^{43}$ Ibíd., 126.

${ }^{44}$ Ibíd.
} 
consecuencias hasta el punto del maleficio y se convierte en víctima del sistema patriarcal.

El tercer movimiento corresponde al viaje de la protagonista y su progenitora a la ciudad, para encontrar una respuesta por parte del presidente y solucionar la manutención obligatoria, pues el padre no se responsabilizaría. En este caso, la única referencia a los cuerpos se da al mencionar que «El Presidente tenía fama de castigar físicamente a los hombres que no se encargaban de sus responsabilidades y protegía a las mujeres para que salieran adelante.»> ${ }^{45}$

En el cuarto movimiento, se da la llegada de los personajes al pueblo y las vicisitudes del recorrido. La madre es nombrada por el presidente como maestra rural y enviada a la aldea El Quebracho. En este lugar, hay una mujer que habita en una choza y su aspecto es extraño, de mirada desorbitada, llena de babas, flaca, con las manos negras, se le compara con un animal. Una vez más, se establece la comparación de un humano con los animales, brindando corporeidades grotescas.

Más adelante describe a la abuela, quien va con ellas hacia el nuevo destino, en este caso, es descrita como un objeto «[...] rígida, recta, callada, como un bulto más sentado a la par del dueño de los bueyes [...]» ${ }^{46}$. Como se observa, la caracterización pasa a ser la adjetivación de una cosa, alguien sin sentimientos, en el silencio de las cavilaciones.

De este modo, los cuatro movimientos ofrecen al lector una serie de corporeidades que oscilan entre la descripción física hasta la animalización de los personajes, especialmente los hombres o las personas de menor nivel social y educativo.

\section{Conclusiones}

A lo largo de este recorrido, de manera breve, se analiza la obra Con pasión absoluta de Carol Zardetto, un texto lleno de reconocimientos y que permite ojear, desde la mirada femenina, la memoria de tres generaciones guatemaltecas.

Analizar, a partir de los paratextos, brinda una visión historiográfica que abarca títulos, epígrafes, notas, epílogos, entre otros, que, por sí mismos, dialogan con el lector, brindan una pauta para la interpretación y, en el caso de la obra de Zardetto, los presenta como un mecanismo para evidenciar cómo la memoria histórica habita en los textos contemporáneos.

45 Ibíd., 127

${ }^{46}$ Ibíd., 132.

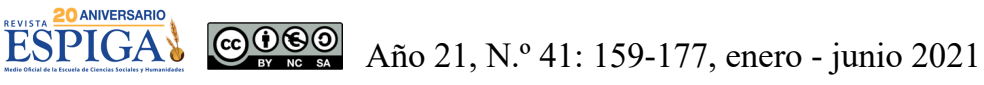


La hibridez en la obra Con pasión absoluta, que de forma coherente va entrelazando recursos tales como el uso de cartas, columnas, cuadros, prosa poética, entre otros, y el contenido textual de dichos apartados; es un indicio de que en los textos centroamericanos actuales se dialoga desde la marginalidad, pues en la novela se escuchan las voces de personajes rechazados, aislados socialmente, quienes tratan de hacerse sentir.

Lo anterior se manifiesta desde el título, que muestra sentimientos, una doble significación, una actitud de desencanto; la dedicatoria, asimismo, establece claramente el público a quien está dirigida la obra: las mujeres y en especial aquellas que son de familia, individuos que aún mantienen en su corporeidad y estatus social. Por último, el capítulo analizado está impregnado de sentimientos movidos por el cuerpo y la marginalidad.

Para finalizar, Zardetto presenta una obra de importancia para los estudios guatemaltecos y latinoamericanos, porque es representación de una sociedad golpeada, sufriente, violentada a lo largo de los siglos y que expresa la relación entre el texto y el contexto que la vio nacer.

\section{Formato de citación según APA}

Zúñiga-Hernández, L. (2021). Cuerpo marginal desde los paratextos literarios de la obra de Con pasión absoluta de Carol Zardetto. Revista Espiga, 21 (41), páginas 159-177.

\section{Formato de citación según Chicago-Deusto}

Zúñiga-Hernández, Laura. «Cuerpo marginal desde los paratextos literarios de la obra de Con pasión absoluta de Carol Zardetto». Revista Espiga 21, n. ${ }^{\circ} 41$ (enero-junio, 2021): páginas 159-177.

\section{Referencias}

Álamo, Francisco. «Paratextualidad y novela: Las partes del texto o el diseño editorial». Anuario de estudios filológicos 32, (2009): 5-21.

Arias, Arturo. Gestos ceremoniales. Narrativa centroamericana 1960-1990. Guatemala: Artemis y Edinter, 1998.

Boubée, Carolina, Olga Graciela Delorenzi y Patricia Sastre Vázquez. «La comprensión: proceso lingüístico y matemático». Revista Iberoamericana de Educación 46, n. ${ }^{\circ} 8$ (2008): 1-9. 
Castro, María de los Ángeles. «La construcción de lo indígena en textos etnoficcionales en Centroamérica». Ístmica, n. ${ }^{\circ} 11$ (2007): 131-154.

Cortez, Beatriz. Estética del cinismo. Pasión y desencanto en la literatura centroamericana de posguerra. Guatemala: F y G Editores, 2010.

Elvir, Lety. Sublimes y perversos. Tegucigalpa: Litografía López, 2005.

Escudos, Jacinta. AB sudario. San Salvador: Alfaguara, 2003.

Fajardo, Luz Amparo. «La metáfora, un recurso en la formación de pensamiento». Instituto de Ciencias de la Educación para la Investigación Interdisciplinaria de la Facultad de Ciencias Humanas, n. ${ }^{\circ} 11$ (2007): 103-111.

Foucault, Michelle. Vigilar y castigar. Nacimiento de la prisión. México: Siglo XXI editores, S.A, 1976.

Gama, Michelle. «El cuerpo marginal: los símbolos del cuerpo en el cuento "El cobrador" de Rubem Fonseca». Mitologías Hoy, n. ${ }^{\circ} 6$ (2014): 48-55.

Gutiérrez Álvarez, Coralia. «La historiografía contemporánea sobre la independencia en Centroamérica». Nuevo Mundo Mundos Nuevos, Debates, 3 febrero 2009. Acceso: 27 de mayo de 2021. http://journals.openedition.org/nuevomundo/54642

Fajardo, Luz. «La metáfora, un recurso en la formación del pensamiento». Praxis, n. ${ }^{\circ} 11(2007)$.

Hernández, Claudia. De fronteras. Guatemala: Editorial Piedra Santa, 2007.

Laín, Guillermo. «Teoría y práctica de la metáfora en torno a Fervor de Buenos Aires, de Borges». Cuadernos de ALEPH, n. 2 (2007): 79-93.

Lakoff, George y Jonhson-Mark. Metáforas de la vida cotidiana. Madrid: Cátedra Teorema, 1995.

Le Breton, David. Antropología del cuerpo y modernidad. Buenos Aires: Ediciones Nueva Visión, 2002.

Maldonado, María del Pilar. «Sobre la memoria cultural e histórica en Guatemala. La obra de Carol Zardetto: Con pasión absoluta». Tesis doctoral. Universität Wien, 2010. Acceso: 27 de mayo de 2021. https://docplayer.es/14309755Diplomarbeit-titel-der-diplomarbeit-sobre-la-memoria-cultural-e-historica-enguatemala-la-obra-de-carol-zardetto-con-pasion-absoluta.html

Paz, José María. «Texto y paratexto en el Quijote». Conferencia pronunciada en Actas II. II Congreso de la Asociación Internacional Siglo de Oro. Centro Cervantes Virtual, Universidad de Salamanca, 1990. Acceso: 3 de junio del 2021. https://cvc.cervantes.es/literatura/aiso/pdf/02/aiso_2_2_030.pdf 
Sabia, Säid. «Paratexto. Títulos, dedicatorias y epígrafes en algunas novelas mexicanas». Espéculo: Revista de estudios literarios, n. ${ }^{\circ} 31$ (2005). Acceso: 27 de mayo de 2021. https://webs.ucm.es/info/especulo/numero31/paratext.html

Santos, Luis. «¿Qué es ser hombre? Reflexiones sobre la masculinidad». En Relación de saberes. Bogotá: Universidad Nacional de Colombia, 1998.

Sesarego, Roberto. «El cuerpo marginal: aspectos de la fenomenología del cuerpo explorada desde Gurwitsch». Conferencia pronunciada en la I Jornadas de Estudiantes Departamento de Filosofía en la Universidad de Buenos Aires, 25 al 28 de noviembre de 2014.

Zardetto, Carol. Con pasión absoluta. Guatemala: F y G Editores, 2005.

Zavala, Magda. Globalización y literatura en América Central: escritores y editoriales. Hacia una historia de las literaturas centroamericanasIntersecciones y trasgresiones: Propuesta para una historiografia literaria en Centroamérica. Guatemala: F y G Editores, 2008. 